\title{
Epigallocatechin gallate suppresses peritoneal fibrosis in mice
}

Mineaki Kitamura $^{1}$, Tomoya Nishino ${ }^{1}$, Yoko Obata ${ }^{1,2}$, Akira Furusu ${ }^{1}$, Yoshitaka Hishikawa ${ }^{3}$, Takehiko Koji ${ }^{3}$, and Shigeru Kohno ${ }^{1}$

${ }^{1}$ Second Department of Internal Medicine, Nagasaki University School of Medicine

${ }^{2}$ Medical Education Development Center, Nagasaki University Hospital

${ }^{3}$ Department of Histology and Cell Biology, Unit of Basic Medical Science, Nagasaki

University Graduate School of Biomedical Sciences

Running title: EGCG in peritoneal fibrosis

Corresponding author: Tomoya Nishino, M.D., Ph.D.

Second Department of Internal Medicine

Nagasaki University School of Medicine

1-7-1 Sakamoto, Nagasaki 852-8501, Japan

Tel: +81-95-819-7273 Fax: +81-95-849-7285

E-mail: tnishino@nagasaki-u.ac.jp 


\begin{abstract}
Long-term peritoneal dialysis (PD) leads to histological changes in the peritoneal membrane.
\end{abstract}

Angiogenesis and inflammation caused by glucose degradation products (GDPs) play crucial roles in peritoneal fibrosis. One such GDP is methylglyoxal (MGO), which enhances the formation of advanced glycation end products (AGEs). AGEs bind to their receptor (RAGE) and activate nuclear factor- $\kappa \mathrm{B}(\mathrm{NF}-\kappa \mathrm{B})$, which is a key regulator of angiogenesis and inflammation. Recent studies have indicated that (-)-epigallocatechin gallate (EGCG), a tea polyphenol, inhibits angiogenesis and inflammation. Here, we examined whether EGCG suppresses peritoneal fibrosis in mice.

Based on preliminary examination, $2 \mathrm{~mL}$ of $40 \mathrm{mM}$ MGO or PD fluid was injected intraperitoneally and EGCG $(50 \mathrm{mg} / \mathrm{kg})$ or saline was injected subcutaneously for 3 weeks. In comparison to PD fluid + saline-treated mice, the peritoneal tissues of MGO + salinetreated mice showed marked thickening of the submesothelial compact zone. In the submesothelial compact zone of the $\mathrm{MGO}+$ saline-treated mice, CD31-positive vessels and vascular endothelial growth factor-positive cells were significantly increased, as were inflammation, F4/80-positive macrophages, and monocyte chemotactic protein-1. Moreover, 8-hydroxydeoxyguanosine, a marker of reactive oxygen species, and NF- $\kappa \mathrm{B}$, determined by 
Southwestern histochemistry, in the submesothelial compact zone were also increased in

$\mathrm{MGO}+$ saline-treated mice. These changes were attenuated in MGO + EGCG-treated mice.

We demonstrated that EGCG treatment suppresses peritoneal fibrosis via inhibition of NF-

$\kappa B$. Furthermore, EGCG inhibits reactive oxygen species production. The results of this

study indicate that EGCG is a potentially novel candidate for the treatment of peritoneal

fibrosis.

Key words: angiogenesis, (-)-epigallocatechin gallate (EGCG), inflammation methylglyoxal

(MGO), peritoneal dialysis (PD), peritoneal fibrosis 


\section{Introduction}

Peritoneal dialysis (PD) is a beneficial therapy for end-stage renal disease. However, longterm PD therapy leads to histopathological changes in the peritoneum, such as peritoneal fibrosis and vasculopathy $[1,2]$. Although the mechanisms underlying these structural changes have not been fully elucidated, the angiogenesis and inflammation caused by the glucose degradation products (GDPs) in PD fluid play crucial roles [3, 4]. Some GDPs, such as methylglyoxal (MGO), promote generation of advanced glycation end products (AGEs) [5-7]. Recent studies have revealed that peritoneal deposition of AGEs increases with the duration of $\mathrm{PD}$, and may correlate with the development of peritoneal fibrosis $[4,6,8]$.

Growing evidence indicates that AGEs evoke angiogenesis through stimulating vascular endothelial growth factor (VEGF), and also induce proinflammatory cytokines $[4,9]$. The interaction of AGEs with the receptor for AGEs (RAGE) results in peritoneal damage in diabetic and uremic rats [10-12], and causes upregulation of the transcription factor, nuclear factor- $\kappa \mathrm{B}(\mathrm{NF}-\kappa \mathrm{B})[9,12,13]$. NF- $\mathrm{kB}$ plays a key role in regulating target genes, such as VEGF, monocyte chemotactic protein-1 (MCP-1), interleukin (IL)-1 $\alpha$, and IL-6 [9].

One of the most common GDPs found in PD fluid is MGO, which enhances the formation of AGEs [7]. Previous investigations of rat MGO-induced peritoneal fibrosis have revealed 
thickening of the peritoneum and ultrafiltration failure; these are major symptoms of peritoneal fibrosis in PD patients [7, 14]. Although several other peritoneal fibrosis models exist, we established a mouse MGO-induced peritoneal fibrosis model in the present study. The mouse offers critical advantages as an experimental model for mammalian biology, including low cost, fast turnover, easy breeding, and multiple possibilities of genetic manipulation.

Although the mechanism underlying MGO-induced peritoneal fibrosis has not been fully elucidated, previous research has indicated that tissue damage with inflammation, subsequently followed by tissue repair with excessive proliferation of myofibroblasts [14], and angiogenesis play important roles $[2,4,15]$. The accumulation of AGEs in the MGOinduced rat peritoneal fibrosis model has been demonstrated [7], and oxidative stress has been shown to play a key role in the production of AGEs, and also in the cell signaling of downstream RAGE [16]. Furthermore, increased NF- $\kappa$ B expression has been observed in infiltrating cells of the submesothelial compact zone, and the degree of submesothelial NF$\kappa \mathrm{B}$ expression was found to correlate significantly with the progression of peritoneal fibrosis in PD patients [13].

Among tea polyphenols, epigallocatechin-3-gallate (EGCG) is known to have favorable 
effects, including the prevention of angiogenesis, inflammation, and oxidation, at least in part, through the suppression of NF- $\mathrm{KB}$ activity [17-19]. Studies using a $\mathrm{CCl}_{4}$-induced liver injury model [20], a bleomycin-induced pulmonary fibrosis model [21], and a cardiac hypertrophy model [22] have demonstrated that EGCG ameliorates fibrosis effectively. Moreover, EGCG significantly decreased AGE-stimulated gene expression [19]. In the present study, we examined the effect of EGCG on peritoneal fibrosis in a mouse MGO-induced model. In addition, we investigated the effects of EGCG on angiogenesis, inflammation, and oxidative stress, to elucidate the inhibitory mechanisms of EGCG for peritoneal fibrosis. 


\section{Materials and methods}

\subsection{Animals}

The animals used in this study were male C57/BL6 mice (10 weeks of age, weighing approximately $25 \mathrm{~g}$; Japan SLC Inc., Shizuoka, Japan). The animals had free access to laboratory chow and tap water, and were housed in a light- and temperature-controlled room in the Biomedical Research Center, Center for Frontier Life Sciences, Nagasaki University School. The experimental protocol was inspected by the Animal Care and Use Committee of Nagasaki University School, and approved by the President of Nagasaki University School (Approval number: 1003301048).

\subsection{Reagents}

PD fluid containing 2.5\% glucose was made by mixing Dianeal ${ }^{\circledR}$ N PD-2 1.5 (Baxter, Tokyo, Japan) and 50\% glucose solution. MGO (155558; MP Biomedicals LLC, Illkirch, France) was dissolved with PD fluid. EGCG (70935; Cayman Chemicals, MI, USA) was dissolved in saline.

Following purification with a $0.2-\mu \mathrm{m}$ pore-size filter, the PD fluids with or without MGO were prepared and adjusted with $\mathrm{HCl}$ daily to $\mathrm{pH}$ 5.0, immediately prior to injection. 
3,3'-diaminobenzidine-4HCl (DAB) and ethylenediaminetetraacetic acid (EDTA) were

purchased from Dojin Chemical (Kumamoto, Japan). Tris-HCl, bovine serum albumin

(BSA), yeast transfer RNA, salmon testis DNA, normal sheep immunoglobulin G (IgG), and

Brij 35 were purchased from Sigma Chemical (St. Louis, MO, USA). All other reagents were purchased from Wako Pure Chemicals (Osaka, Japan).

\subsection{Antibodies}

The following first antibodies were used for immunohistochemistry: (1) mouse anti-

carboxy methyllysine (CML) (one of AGEs derived from MGO) antibody, diluted 1/50

(KH011; Dojin Chemical); (2) rabbit anti-RAGE antibody, diluted 1/800 (AB5484; Millipore,

CA, USA); (3) rabbit anti-type III collagen antibody, diluted 1/400 (LB-1393; LSL Co.,

Tokyo, Japan); (4) mouse anti- $\alpha$-smooth muscle actin ( $\alpha$-SMA) antibody, diluted 1/100

(A2547; Sigma), used as a marker of myofibroblasts; (5) rabbit anti- transforming growth

factor- $\beta$ (TGF- $\beta$ ) antibody, diluted 1/100 (sc-146; Santa Cruz, CA, USA); (6) mouse anti-8-

hydroxydeoxyguanosine (8-OHdG) antibody, diluted 1/100 (MOG-100P; Japan Institute for

the Control of Aging, Tokyo, Japan), used as a marker of oxidative stress; (7) rat anti-mouse

CD31/PECAM-1 antibody, diluted 1/50 (sc-1506; Santa Cruz), used as a marker of 
endothelial cells; (8) rabbit anti-VEGF antibody, diluted 1/100 (sc-152; Santa Cruz); (9) rat anti-F4/80 antibody, diluted 1/50 (MCA497; Serotec, Oxford, UK), used as a marker of mouse macrophages; (10) goat anti-mouse MCP-1 antibody, diluted 1/100 (sc-1784; Santa Cruz); and (11) sheep anti- digoxigenin (DIG) horseradish peroxidase (HRP)-conjugated sheep antibody (11207733910; Roche, Mannheim, Germany).

The following antibodies were used as second or third antibodies: (1) HRP-conjugated swine anti-rabbit IgG antibody (P0399; Dako, Glostrup, Denmark); (2) The peroxidaseantiperoxidase reaction (PAP, Z0113; Dako); (3) HRP-conjugated rabbit anti-rat IgG antibody (Z0147; Dako); (4) HRP-conjugated goat anti-mouse IgG antibody (P0447; Dako).

\subsection{Administration of PD fluid and EGCG}

Mice were divided into 4 groups: (1) PD fluid + saline group $(n=6)$, defined as the control group; (2) PD fluid + EGCG group $(n=4)$; (3) PD fluid + MGO + saline group $(n=6)$, defined as the MGO group; and (4) PD fluid + MGO + EGCG group ( $n=6)$, defined as the EGCG group. Mice received PD fluid (100 mL/kg) with or without dissolved MGO (40 mM) intraperitoneally for 15 days, 5 consecutive days per week as described previously [7]. Mice also received saline or $50 \mathrm{mg} / \mathrm{kg}$ EGCG in saline subcutaneously for 21 days daily. The dose 
and interval for MGO injection were selected based on pilot studies.

Mice were sacrificed at day 22. The peritoneal tissues were dissected out and fixed in $4 \%$ paraformaldehyde in phosphate-buffered saline (PBS) (pH 7.4).

\subsection{Histological analysis and immunohistochemistry}

For histrological examination, 4- $\mu \mathrm{m}$-thick paraffin-embedded tissues were stained using the Masson-trichrome method.

An indirect immunohistochemical technique was used for CML, type III collagen, $\alpha$-SMA, 8-OHdG, and F4/80. The indirect staining was performed as described previously [15, 23]. In brief, deparaffinized tissue sections were incubated for 30 min with a blocking buffer containing 5\% normal goat serum, 5\% fetal calf serum, $5 \%$ BSA, and 20\% normal swine serum in PBS. The sections were then reacted with the primary antibodies, and diluted in the same blocking buffer. Following reaction with anti-type III collagen antibody for $1 \mathrm{~h}$ at room temperature, sections were reacted with HRP-conjugated swine anti-rabbit immunoglobulin antibody diluted $1 / 50$, for $30 \mathrm{~min}$ at room temperature. The peroxidase-antiperoxidase reaction diluted 1/100 was used. For F4/80, sections were reacted with HRP-conjugated rabbit anti-rat immunoglobulin antibody and HRP-conjugated swine anti-rabbit 
immunoglobulin antibody, following reaction with anti-F4/80 antibody for $1 \mathrm{~h}$ at room

temperature. For CML, $\alpha-\mathrm{SMA}$, and $8-\mathrm{OHdG}$, sections were reacted with primary

antibodies overnight at room temperature, and HRP-conjugated goat anti-mouse

immunoglobulin antibody diluted $1 / 50$, for $1 \mathrm{~h}$ at room temperature.

For RAGE, TGF- $\beta$, CD31, VEGF, and MCP-1, tissues were stained by an avidin-biotin

complex technique using a Vectastain Elite ABC kit (Vector Laboratories, CA, USA),

following reaction with the primary antibody as described previously [15, 23]. In brief, all

primary antibodies except for MCP-1 were reacted for $1 \mathrm{~h}$ at room temperature. For MCP-1, the primary antibody was reacted overnight at $4^{\circ} \mathrm{C}$.

Reaction products were visualized by treating sections with $100 \mu \mathrm{L}$ of $30 \%$ hydrogen

peroxide and $50 \mathrm{mg}$ of DAB. Finally, the sections were counterstained with methyl green and mounted.

\subsection{Southwestern histochemistry for $N F-\kappa B$}

The following single-strand (ss)-oligo-DNAs were synthesized with the specific consensus sequence for the NF- $\mathrm{BB}$ binding site (5'-GATCGAGGGGGACTTTCCCTAGC-3' and 5'GCTAGGGAAAGTCCCCCTCGATC-3') [24]. Double-strand (ds)-oligo-DNA was 3'- 
labeled with DIG. The synthesized ss-oligo-DNAs were purchased from BEX (Tokyo, Japan).

For annealing of complementary ss-oligo-DNAs to ds-oligo-DNA, the ss-oligo-DNAs were dissolved in $10 \mathrm{mmol} / \mathrm{L}$ of Tris- $\mathrm{HCl}$ buffer $(\mathrm{pH} 7.4)$, denatured for $10 \mathrm{~min}$ at $100^{\circ} \mathrm{C}$, and gradually cooled to room temperature.

Southwestern histochemistry was performed as described previously [25-27]. In brief, deparaffinized sections (4- $\mu$ m-thick) were treated in a microwave oven with citrate buffer $(\mathrm{pH} 6.0)$ at $95^{\circ} \mathrm{C}$ for $15 \mathrm{~min}$. The sections were then washed in PBS and immersed in a preincubation buffer containing 5\% nonfat milk dissolved in $10 \mathrm{mmol} / \mathrm{L}$ of Tris- $\mathrm{HCl}(\mathrm{pH} 7.4)$ with $300 \mathrm{mmol} / \mathrm{L}$ of $\mathrm{NaCl}$ and $1 \mathrm{mmol} / \mathrm{L}$ of EDTA for $1 \mathrm{~h}$. Next, the sections were incubated overnight with the probe dissolved in the pre-incubation buffer at a concentration of $1 \mu \mathrm{g} / \mathrm{mL}$. The sections were then washed twice with pre-incubation buffer, and 4 times with $0.075 \%$ Brij in PBS. Then the sections were incubated in a blocking buffer containing 5\% BSA, 500 $\mu \mathrm{g} / \mathrm{mL}$ of normal sheep IgG, $100 \mu \mathrm{g} / \mathrm{mL}$ of yeast transfer RNA, and $100 \mu \mathrm{g} / \mathrm{mL}$ of salmon sperm DNA in PBS for $1 \mathrm{~h}$. To visualize hybridized DNA-oligo probes, the sections were immunohistochemically stained with anti-DIG HRP-conjugated sheep antibody, diluted 1/100 in blocking buffer, for $2 \mathrm{~h}$. After 4 washes with $0.075 \%$ Brij in PBS, we performed a color reaction with $100 \mathrm{mmol} / \mathrm{L}$ of phosphate buffer ( $\mathrm{pH} 7.6$ ), containing $50 \mathrm{mg}$ of DAB and $33 \mu \mathrm{L}$ 
of $30 \%$ hydrogen peroxide. To enhance the reaction, we added $2 \mathrm{~mL}$ of $0.5 \%(\mathrm{v} / \mathrm{v})$ nickel ammonium sulfate solution and $2.5 \mathrm{~mL}$ of $0.5 \%(\mathrm{v} / \mathrm{v})$ cobalt chloride solution. To evaluate the specificity of the probe for NF- $\kappa \mathrm{B}$, we also used a mutated probe $\left(5^{\prime}-\right.$ GATCGAGGAAGACTTTCCCTAGC-3' and 5'-GCTAGGGAAAGTCTTCCTCGATC-3')

[24]. Moreover, we hybridized some sections with the probe in the presence of an excess amount of non-labeled homologous ds-oligo-DNA, as described previously [26, 27].

\subsection{Morphometric analysis}

To assess the thickening of the submesothelial compact zone, we used image analysis software (Lumina Vision Ver. 2.04; Mitani Corp., Fukui, Japan) following Masson-trichrome staining. We measured the thickness of the submesothelial compact zone at 5 randomly selected positions in each tissue at $\times 200$ magnification, and averaged the results.

In each sample, we counted the numbers of RAGE-expressing cells, $\alpha$-SMA-expressing cells, TGF- $\beta$-expressing cells, 8-OHdG-expressing cells, CD31-positive vessels, VEGFexpressing cells, F4/80-positive macrophages, and MCP-1-expressing cells in 5 fields at $\times 200$ magnification. The results for each tissue were averaged. For CML and type III collagen, we selected 5 areas in each tissue and performed semi-quantitative estimation of the intensity of 
each protein with Lumina Vision.

Finally, we selected 5 areas in each tissue and evaluated NF-кB-activated cells with Lumina Vision.

\subsection{Statistical analysis}

Data are expressed as mean \pm standard error of the mean (SEM). Differences among groups were examined for statistical significance using repeated measures analysis of variance

(ANOVA) (Bonferroni/Dunn test). A $p$ value $<0.05$ denotes a statistically significant difference. All statistical analyses were performed with Stat View version 5.0 software (SAS Institute Inc., NC, USA). 


\section{RESULTS}

\subsection{Morphological examination following Masson-trichrome staining}

In the normal and control mice, the peritoneal tissue consisted of a peritoneal mesothelial monolayer and sparse connective tissues below this layer (Fig. 1A and B). Compared to the control group, the peritoneal tissue of the MGO group showed significant thickening of the submesothelial compact zone and the presence of numerous cells (Fig. 1C and F). The thickness of the submesothelial compact zone in the EGCG group was significantly lower than that of the MGO group (Fig. 1D and F). To assess the toxicity of EGCG, we analyzed the PD fluid + EGCG group. Repeated injections of EGCG had no significant effect on the peritoneum (Fig. 1E and F).

\subsection{Accumulation of CML and expression of RAGE}

We observed very little CML deposition in the peritoneal tissues of the control group (Fig.

2A and D). The MGO group showed intense staining of CML in the peritoneum (Fig. 2B and D), whereas the EGCG group showed reduced intensity of CML staining (Fig. 2C and D).

The number of RAGE-expressing cells was significantly higher in the MGO group than in the control group (Fig. 2E, F, and H), but significantly lower in the EGCG group than in the 
MGO group (Fig. 2F, G, and H).

\subsection{Expression of type III collagen, $\alpha$-SMA, and TGF- $\beta$}

In the control group, type III collagen expression in the submesothelial compact zone was equivalent to that of normal mice (Fig. 3A). In the MGO group, type III collagen was diffusely expressed, and the positive areas were significantly larger than those of the control group (Fig. 3B and D). EGCG administration significantly reduced the expression of type III collagen (Fig. 3C and D).

Previous studies have shown that peritoneal myofibroblasts and mesothelial cells produce collagen, and that collagen deposition is mainly due to myofibroblasts $[28,29]$. Thus, we investigated $\alpha$-SMA-expressing cells as a marker of myofibroblasts. In the MGO group, we observed the expression of $\alpha$-SMA in vascular smooth muscle cells, and also numerous $\alpha$ SMA-expressing myofibroblasts (Fig. 4A and Table). On the contrary, the EGCG group contained markedly fewer $\alpha$-SMA-positive myofibroblasts (Fig. 4B and Table). We also investigated fibroblast specific marker-1 (FSP-1) as an immunohistochemical marker of fibroblasts and myofibroblasts. The number of FSP-1 positive cells was greater than that of $\alpha$-SMA positive cells in the submesothelial compact zone (data not shown). This suggested 
that not only myofibroblasts but also fibroblasts were present in the peritoneal tissue.

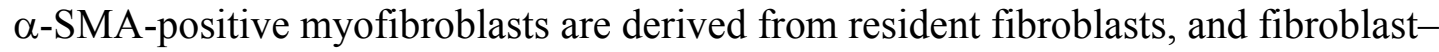

myofibroblast differentiation is mainly mediated by TGF- $\beta$ [30]. Therefore, we also

evaluated the levels of TGF- $\beta$-expressing cells. In the MGO group, the number of TGF- $\beta$ -

expressing cells was higher than that of the control group (Fig. 4C and Table). However, the

EGCG group contained fewer TGF- $\beta$-expressing cells (Fig. 4D and Table).

\subsection{Expression of 8-OHdG}

EGCG is known to possess an anti-oxidative effect [17]. Therefore, we evaluated 8-OHdG

as a marker of oxidative stress. We found that $8-\mathrm{OHdG}$-expressing cells were mainly located

in the submesothelial compact zone. However, the numbers of 8-OHdG-expressing cells

differed significantly between the groups. In the control group, the number of $8-\mathrm{OHdG}-$

expressing cells was slightly higher than that of normal mice (data not shown). In the MGO

group, the number of 8-OHdG-expressing cells was higher than that of the control group (Fig.

4E and Table). Meanwhile, the EGCG group contained fewer 8-OHdG-expressing cells (Fig.

4F and Table). 


\subsection{Expression of CD31 and VEGF}

We investigated angiogenesis in peritoneal fibrosis, and quantified the number of CD31-

positive blood vessels. In the MGO group, the number of blood vessels was markedly higher than in the control group (Fig. 5A and Table). However, the EGCG group contained fewer blood vessels (Fig. 5B and Table).

VEGF is a potent stimulator of angiogenesis. Therefore, we evaluated VEGF in the peritoneal tissue. In the MGO group, the number of VEGF-expressing cells was markedly higher than in the control group (Fig. 5C and Table). However, the EGCG group contained fewer VEGF-expressing cells in the submesothelial zone (Fig. 5D and Table).

\subsection{Expression of $\mathrm{F} 4 / 80$ and $M C P-1$}

To investigate inflammation, we stained sections with anti-F4/80 antibody, as a marker of macrophages. In the MGO group, we observed numerous macrophages in the thickened peritoneal tissues (Fig. 5E and Table). However, the EGCG group contained significantly fewer macrophages than did the MGO group (Fig. 5F).

We also investigated the levels of MCP-1-expressing cells, which exert an important effect on macrophage infiltration. In the MGO group, the number of MCP-1-expressing cells was 
significantly higher than that in the control group (Fig. 5G and Table). Compared to the MGO group, the EGCG group contained significantly fewer MCP-1-expressing cells (Fig. $5 \mathrm{H}$ and Table).

\subsection{Southwestern histochemistry for $N F-\kappa B$}

EGCG has been shown to suppress NF-אB signaling [31, 32]. Therefore, we investigated the expression of activated NF-кB in our model. Southwestern histochemistry is used to detect activated transcription regulatory factors that bind to specific sequences of DNA [25, 26].

The number of NF- $\mathrm{kB}$-activated cells in the submesothelial compact zone of the MGO group was significantly higher than that of the control group (Fig. 6A, B, and D). Compared

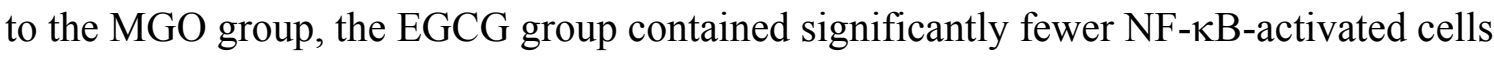
(Figure 6C and D). To evaluate the specificity, we performed the same procedure using a mutated probe. In this case, only a weak nuclear staining for NF- $\kappa \mathrm{B}$ was detected (data not shown). When an excess amount of non-labeled probe was added to the standard mixture containing DIG-labeled probe, absent or weak nuclear staining for NF- $\kappa \mathrm{B}$ was detected (data not shown). 


\section{Discussion}

In the present study, MGO administration markedly increased the thickness of the

submesothelial compact zone, the number of blood vessels and macrophages, and the intensity of immunohistochemical staining for CML in the mouse peritoneum. Moreover, mice died from colon adhesion and we observed the formation of abdominal cocoons (data not shown). All of these changes, reflecting the features of human peritoneal fibrosis, were significantly suppressed in the EGCG group. Our results strongly indicate that EGCG ameliorates peritoneal fibrosis.

The underlying mechanisms of encapsulating peritoneal sclerosis (EPS) in humans are not fully understood. With respect to vasculopathy in the MGO-induced peritoneal fibrosis model, the number of vessels increased in the MGO group, which is similar to human peritoneal fibrosis [1]. However, it was difficult to evaluate morphological changes in the vessels because of the small size of the vessels in mice. In the perineum of patients on longterm peritoneal dialysis, vasculopathy tends to occur in post-capillary venules or capillaries and is marked by stenosis or obstruction of the vascular lumen caused by thickening and hyalinization of the vessels $[1,33]$.

Although structural changes could not be evaluated in detail, we found no intraluminal 
stenosis or obstruction in our peritoneal fibrosis model. Vasculopathy in this peritoneal fibrosis model may be somewhat different from that of human peritoneal fibrosis. One possible explanation for this may be the different time course and contributing factors to the development of fibrosis in humans and the mouse model.

Not limited to vasculopathy, it is difficult to establish an equivalent animal model for EPS. However, peritoneal fibrosis is essential to the development of EPS [4], and therefore, the prevention of peritoneal fibrosis is important to avoid EPS. Although chlorhexidine gluconate -induced peritoneal fibrosis is a well-established animal model, its principal mechanism is chemical irritation of mesothelial cells. By contrast, GDPs are thought to induce peritoneal fibrosis in humans, and therefore, MGO-induced peritoneal fibrosis more closely resembles human peritoneal fibrosis [34]. Several researchers have used a rat MGOinduced peritoneal fibrosis model $[7,14,34]$. However, to our knowledge, this is the first study to use a mouse MGO-induced peritoneal fibrosis model. The rat MGO models are limited by lack of specificity, and transient efficacy of pharmacologic agents and expression protocols. Genetically modified mice could provide an attractive alternative to such models. The concentrations of MGO used in the rat MGO-induced peritoneal fibrosis model [7, 14, 34] were $<20 \mathrm{mM}$. However, in mice, we were unable to induce sufficient peritoneal 
thickness using $20 \mathrm{mM}$ MGO. This may reflect a species-dependent difference. Thus, we adopted the experimental protocol described in the Materials and methods.

Accumulated evidence indicates that GDPs can react with protein and generate AGEs, which are known to be important precursors of peritoneal fibrosis. The extent of AGEs and RAGE expression in the peritoneal submesothelial compact zone has been shown to correlate with the duration of PD therapy $[8,13]$. In general, RAGE is expressed in a variety of cell types, including endothelial cells, vascular smooth muscle cells, macrophages, mesangial cells, and neurons [35]. RAGE expression is low during homeostasis, but strikingly enhanced in conditions such as diabetes, uremia, and inflammation [10]. The AGEs-RAGE interaction activates numerous signaling cascades, including the MAPK cascade and NF- $\mathrm{KB}$ signaling, and plays an important role in peritoneal fibrosis. The interaction also mediates the generation of reactive oxygen species (ROS), which in turn activate TGF- $\beta$-Smad signaling $[12,16]$. In the present study, we observed an increase in TGF- $\beta$-positive cells, and also in 8OHdG-positive cells, following the administration of MGO. Our results suggest that the generation of ROS may increase the activity of TGF- $\beta$.

We have demonstrated for the first time that EGCG attenuates MGO-induced peritoneal fibrosis. Previous reports have revealed that EGCG inhibits the nuclear translocation of NF- 
$\kappa \mathrm{B}[36]$, the phosphorylating activity of inhibitory $\kappa \mathrm{B}$ kinase $-\beta[37]$, and the phosphorylation

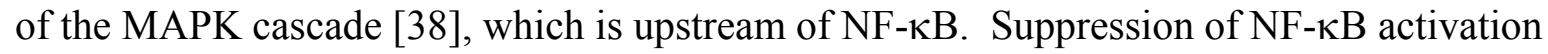
has been linked with anti-inflammatory and anti-angiogenesis activity. Thus, we postulated that EGCG exerts its inhibitory effects on MCP-1 and VEGF expression, at least in part,

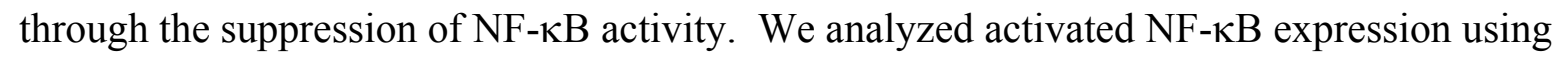
Southwestern histochemistry and observed that the levels of NF- $\kappa \mathrm{B}$ activation correlated with the progression of peritoneal thickness, and also with the expression levels of MCP-1 and VEGF.

EGCG should be administrated intraperitoneally, intravenously, or subcutaneously, while considering the involvement of liver metabolism and glucuronidation of EGCG following oral administration $[39,40]$. For intraperitoneal injection, direct interaction between EGCG and MGO should be considered. In addition, repeated intravenous injections were technically difficult and created the potential for procedural artifacts in our study. According to the literature, the bioavailability of subcutaneously injected EGCG was superior to that of the orally administered drug [41]. Thus, we adopted the subcutaneous injection of EGCG as the route of administration.

We considered the possibility that EGCG pharmacologically inhibits MGO since Wu et al. 
showed that EGCG prevented production of AGEs in vitro [42]. Therefore, we adopted different EGCG and MGO administration methods to minimize the impact of EGCG on MGO-derived AGE production in our study. To clarify the precise mechanisms of EGCG prevention of MGO-induced peritoneal fibrosis, further investigations will be needed.

The amount of EGCG used in our study was almost 10 times higher than that contained in daily drinking tea [43]. A previous report demonstrated that daily administration of 200 $\mathrm{mg} / \mathrm{kg}$ EGCG did not induce toxicity in rats during short-term observation [44]. In our pathophysiological investigation, there was no difference in the liver and kidney tissues of the PD fluid + EGCG and control groups. Moreover, there was no difference in serum creatinine levels at 3 weeks (data not shown). However, according to the reference, long-term and highdose EGCG administration can induce liver toxicity [45]. The appropriate quantity and method of administration for humans, and the long-term toxicity should be examined prior to clinical application.

In conclusion, we have shown for the first time that EGCG inhibits the progression of peritoneal fibrosis in a mouse MGO-induced peritoneal fibrosis model. Our results indicate that EGCG inhibits AGEs-induced upregulation of MCP-1 and VEGF, via inhibition of NF$\kappa \mathrm{B}$ activation and ROS generation. Thus, EGCG represents a potentially novel candidate for 
the treatment of peritoneal fibrosis. 


\section{Conflict of interest}

The author declares no conflict of interest.

\section{Acknowledgment}

This study was supported by grants from the Japanese Association of Dialysis Physicians

(22051). We would like to thank Ms. Ryoko Yamamoto for excellent experimental assistance. 


\section{References}

[1] J.D. Williams, K.J. Craig, N. Topley, C. Von Ruhland, M. Fallon, G.R. Newman, R.K. Mackenzie, G.T. Williams, Morphologic changes in the peritoneal membrane of patients with renal disease, J Am Soc Nephrol 13(2) (2002) 470-479.

[2] V.C. Gandhi, H.M. Humayun, T.S. Ing, J.T. Daugirdas, V.R. Jablokow, S. Iwatsuki, W.P. Geis, J.E. Hano, Sclerotic thickening of the peritoneal membrane in maintenance peritoneal dialysis patients, Archives of internal medicine 140(9) (1980) 1201-1203.

[3] S. Shaw, M. Akyol, J. Bell, J.D. Briggs, M.H. Dominiczak, Effects of continuous ambulatory peritoneal dialysis and kidney transplantation on advanced glycation endproducts in the skin and peritoneum, Cellular and molecular biology (Noisy-le-Grand, France) 44(7) (1998) 1061-1068.

[4] E. Boulanger, N. Grossin, M.P. Wautier, R. Taamma, J.L. Wautier, Mesothelial RAGE activation by AGEs enhances VEGF release and potentiates capillary tube formation, Kidney international 71(2) (2007) 126-133.

[5] T. Oya, N. Hattori, Y. Mizuno, S. Miyata, S. Maeda, T. Osawa, K. Uchida, Methylglyoxal modification of protein. Chemical and immunochemical characterization of methylglyoxal-arginine adducts, The Journal of biological chemistry 274(26) (1999) 18492- 
18502.

[6] K. Honda, K. Nitta, S. Horita, W. Yumura, H. Nihei, R. Nagai, K. Ikeda, S. Horiuchi, Accumulation of advanced glycation end products in the peritoneal vasculature of continuous ambulatory peritoneal dialysis patients with low ultra-filtration, Nephrol Dial Transplant 14(6) (1999) 1541-1549.

[7] M. Nakayama, A. Sakai, M. Numata, T. Hosoya, Hyper-vascular change and formation of advanced glycation endproducts in the peritoneum caused by methylglyoxal and the effect of an anti-oxidant, sodium sulfite, American journal of nephrology 23(6) (2003) 390-394.

[8] M. Nakayama, Y. Kawaguchi, K. Yamada, T. Hasegawa, K. Takazoe, N. Katoh, H. Hayakawa, N. Osaka, H. Yamamoto, A. Ogawa, H. Kubo, T. Shigematsu, O. Sakai, S. Horiuchi, Immunohistochemical detection of advanced glycosylation end-products in the peritoneum and its possible pathophysiological role in CAPD, Kidney international 51(1) (1997) 182-186.

[9] A. Goldin, J.A. Beckman, A.M. Schmidt, M.A. Creager, Advanced glycation end products: sparking the development of diabetic vascular injury, Circulation 114(6) (2006) 597-605. 
[10] A.S. De Vriese, A. Flyvbjerg, S. Mortier, R.G. Tilton, N.H. Lameire, Inhibition of the interaction of AGE-RAGE prevents hyperglycemia-induced fibrosis of the peritoneal membrane, J Am Soc Nephrol 14(8) (2003) 2109-2118.

[11] S.H. Park, E.G. Lee, I.S. Kim, Y.J. Kim, D.K. Cho, Y.L. Kim, Effect of glucose degradation products on the peritoneal membrane in a chronic inflammatory infusion model of peritoneal dialysis in the rat, Perit Dial Int 24(2) (2004) 115-122.

[12] S. Yamagishi, T. Matsui, K. Nakamura, H. Inoue, M. Takeuchi, S. Ueda, K. Fukami, S. Okuda, T. Imaizumi, Olmesartan blocks advanced glycation end products (AGEs)-induced angiogenesis in vitro by suppressing receptor for AGEs (RAGE) expression, Microvascular research 75(1) (2008) 130-134.

[13] L.P. Kihm, D. Wibisono, S. Muller-Krebs, F. Pfisterer, C. Morath, M.L. Gross, M. Morcos, Y. Seregin, A. Bierhaus, P.P. Nawroth, M. Zeier, V. Schwenger, RAGE expression in the human peritoneal membrane, Nephrol Dial Transplant 23(10) (2008) 3302-3306.

[14] I. Hirahara, E. Kusano, S. Yanagiba, Y. Miyata, Y. Ando, S. Muto, Y. Asano, Peritoneal injury by methylglyoxal in peritoneal dialysis, Perit Dial Int 26(3) (2006) 380-392.

[15] Y. Yoshio, M. Miyazaki, K. Abe, T. Nishino, A. Furusu, Y. Mizuta, T. Harada, Y. Ozono, T. Koji, S. Kohno, TNP-470, an angiogenesis inhibitor, suppresses the progression of 
peritoneal fibrosis in mouse experimental model, Kidney international 66(4) (2004) 16771685.

[16] K. Fukami, S. Ueda, S. Yamagishi, S. Kato, Y. Inagaki, M. Takeuchi, Y. Motomiya, R.

Bucala, S. Iida, K. Tamaki, T. Imaizumi, M.E. Cooper, S. Okuda, AGEs activate mesangial TGF-beta-Smad signaling via an angiotensin II type I receptor interaction, Kidney international 66(6) (2004) 2137-2147.

[17] N. Khan, F. Afaq, M. Saleem, N. Ahmad, H. Mukhtar, Targeting multiple signaling pathways by green tea polyphenol (-)-epigallocatechin-3-gallate, Cancer research 66(5) (2006) 2500-2505.

[18] N. Khan, H. Mukhtar, Multitargeted therapy of cancer by green tea polyphenols, Cancer letters 269(2) (2008) 269-280.

[19] Z. Rasheed, A.N. Anbazhagan, N. Akhtar, S. Ramamurthy, F.R. Voss, T.M. Haqqi, Green tea polyphenol epigallocatechin-3-gallate inhibits advanced glycation end productinduced expression of tumor necrosis factor-alpha and matrix metalloproteinase-13 in human chondrocytes, Arthritis research \& therapy 11(3) (2009) R71.

[20] Y. Yasuda, M. Shimizu, H. Sakai, J. Iwasa, M. Kubota, S. Adachi, Y. Osawa, H. Tsurumi, Y. Hara, H. Moriwaki, (-)-Epigallocatechin gallate prevents carbon tetrachloride- 
induced rat hepatic fibrosis by inhibiting the expression of the PDGFRbeta and IGF-1R,

Chemico-biological interactions 182(2-3) (2009) 159-164.

[21] N. Sriram, S. Kalayarasan, G. Sudhandiran, Epigallocatechin-3-gallate exhibits antifibrotic effect by attenuating bleomycin-induced glycoconjugates, lysosomal hydrolases and ultrastructural changes in rat model pulmonary fibrosis, Chemico-biological interactions 180(2) (2009) 271-280.

[22] H.L. Li, Y. Huang, C.N. Zhang, G. Liu, Y.S. Wei, A.B. Wang, Y.Q. Liu, R.T. Hui, C. Wei, G.M. Williams, D.P. Liu, C.C. Liang, Epigallocathechin-3 gallate inhibits cardiac hypertrophy through blocking reactive oxidative species-dependent and -independent signal pathways, Free radical biology \& medicine 40(10) (2006) 1756-1775.

[23] T. Nishino, M. Miyazaki, K. Abe, A. Furusu, Y. Mishima, T. Harada, Y. Ozono, T. Koji, S. Kohno, Antisense oligonucleotides against collagen-binding stress protein HSP47 suppress peritoneal fibrosis in rats, Kidney international 64(3) (2003) 887-896.

[24] Y. Tan, J.S. Zhang, L. Huang, Codelivery of NF-kappaB decoy-related oligodeoxynucleotide improves LPD-mediated systemic gene transfer, Mol Ther 6(6) (2002) $804-812$

[25] T. Koji, K. Komuta, M. Nozawa, S. Yamada, P.K. Nakane, Localization of cyclic 
adenosine 3',5'-monophosphate-responsive element (CRE)-binding proteins by southwestern histochemistry, J Histochem Cytochem 42(10) (1994) 1399-1405.

[26] Y. Hishikawa, E. Damavandi, S. Izumi, T. Koji, Molecular histochemical analysis of estrogen receptor alpha and beta expressions in the mouse ovary: in situ hybridization and Southwestern histochemistry, Med Electron Microsc 36(2) (2003) 67-73.

[27] M. Ashizawa, M. Miyazaki, K. Abe, A. Furusu, H. Isomoto, T. Harada, Y. Ozono, H. Sakai, T. Koji, S. Kohno, Detection of nuclear factor-kappaB in IgA nephropathy using Southwestern histochemistry, Am J Kidney Dis 42(1) (2003) 76-86.

[28] F. Perfumo, P. Altieri, M.L. Degl'Innocenti, G.M. Ghiggeri, G. Caridi, A. Trivelli, R. Gusmano, Effects of peritoneal effluents on mesothelial cells in culture: cell proliferation and extracellular matrix regulation, Nephrol Dial Transplant 11(9) (1996) 1803-1809.

[29] Y. Mishima, M. Miyazaki, K. Abe, Y. Ozono, K. Shioshita, Z. Xia, T. Harada, T. Taguchi, T. Koji, S. Kohno, Enhanced expression of heat shock protein 47 in rat model of peritoneal fibrosis, Perit Dial Int 23(1) (2003) 14-22.

[30] A. Leask, Potential therapeutic targets for cardiac fibrosis: TGFbeta, angiotensin, endothelin, CCN2, and PDGF, partners in fibroblast activation, Circulation research 106(11) (2010) 1675-1680. 
[31] I. Ishida, C. Kohda, Y. Yanagawa, H. Miyaoka, T. Shimamura, Epigallocatechin gallate suppresses expression of receptor activator of NF-kappaB ligand (RANKL) in Staphylococcus aureus infection in osteoblast-like NRG cells, Journal of medical microbiology 56(Pt 8) (2007) 1042-1046.

[32] S.J. Kim, H.J. Jeong, K.M. Lee, N.Y. Myung, N.H. An, W.M. Yang, S.K. Park, H.J. Lee, S.H. Hong, H.M. Kim, J.Y. Um, Epigallocatechin-3-gallate suppresses NF-kappaB activation and phosphorylation of p38 MAPK and JNK in human astrocytoma U373MG cells, The Journal of nutritional biochemistry 18(9) (2007) 587-596.

[33] M.A. Mateijsen, A.C. van der Wal, P.M. Hendriks, M.M. Zweers, J. Mulder, D.G. Struijk, R.T. Krediet, Vascular and interstitial changes in the peritoneum of CAPD patients with peritoneal sclerosis, Perit Dial Int 19(6) (1999) 517-525.

[34] I. Hirahara, Y. Ishibashi, S. Kaname, E. Kusano, T. Fujita, Methylglyoxal induces peritoneal thickening by mesenchymal-like mesothelial cells in rats, Nephrol Dial Transplant 24(2) (2009) 437-447.

[35] J.L. Wautier, P.J. Guillausseau, Diabetes, advanced glycation endproducts and vascular disease, Vascular medicine (London, England) 3(2) (1998) 131-137.

[36] M. Nomura, W. Ma, N. Chen, A.M. Bode, Z. Dong, Inhibition of 12-O- 
tetradecanoylphorbol-13-acetate-induced NF-kappaB activation by tea polyphenols, (-)epigallocatechin gallate and theaflavins, Carcinogenesis 21(10) (2000) 1885-1890.

[37] F. Afaq, V.M. Adhami, N. Ahmad, H. Mukhtar, Inhibition of ultraviolet B-mediated activation of nuclear factor kappaB in normal human epidermal keratinocytes by green tea Constituent (-)-epigallocatechin-3-gallate, Oncogene 22(7) (2003) 1035-1044.

[38] Z. Dong, Effects of food factors on signal transduction pathways, BioFactors (Oxford, England) 12(1-4) (2000) 17-28.

[39] T. Walle, Absorption and metabolism of flavonoids, Free radical biology \& medicine 36(7) (2004) 829-837.

[40] J.D. Lambert, M.J. Lee, H. Lu, X. Meng, J.J. Hong, D.N. Seril, M.G. Sturgill, C.S. Yang, Epigallocatechin-3-gallate is absorbed but extensively glucuronidated following oral administration to mice, The Journal of nutrition 133(12) (2003) 4172-4177.

[41] J.D. Lambert, D.H. Kim, R. Zheng, C.S. Yang, Transdermal delivery of (-)epigallocatechin-3-gallate, a green tea polyphenol, in mice, The Journal of pharmacy and pharmacology 58(5) (2006) 599-604.

[42] C.H. Wu, G.C. Yen, Inhibitory effect of naturally occurring flavonoids on the formation of advanced glycation endproducts, Journal of agricultural and food chemistry 
53(8) (2005) 3167-3173.

[43] H. Mukhtar, N. Ahmad, Tea polyphenols: prevention of cancer and optimizing health, The American journal of clinical nutrition 71(6 Suppl) (2000) 1698S-1702S; discussion 1703S-1694S.

[44] R.A. Isbrucker, J.A. Edwards, E. Wolz, A. Davidovich, J. Bausch, Safety studies on epigallocatechin gallate (EGCG) preparations. Part 3: teratogenicity and reproductive toxicity studies in rats, Food Chem Toxicol 44(5) (2006) 651-661.

[45] J.D. Lambert, M.J. Kennett, S. Sang, K.R. Reuhl, J. Ju, C.S. Yang, Hepatotoxicity of high oral dose (-)-epigallocatechin-3-gallate in mice, Food Chem Toxicol 48(1) (2010) 409416. 


\section{Figure legends}
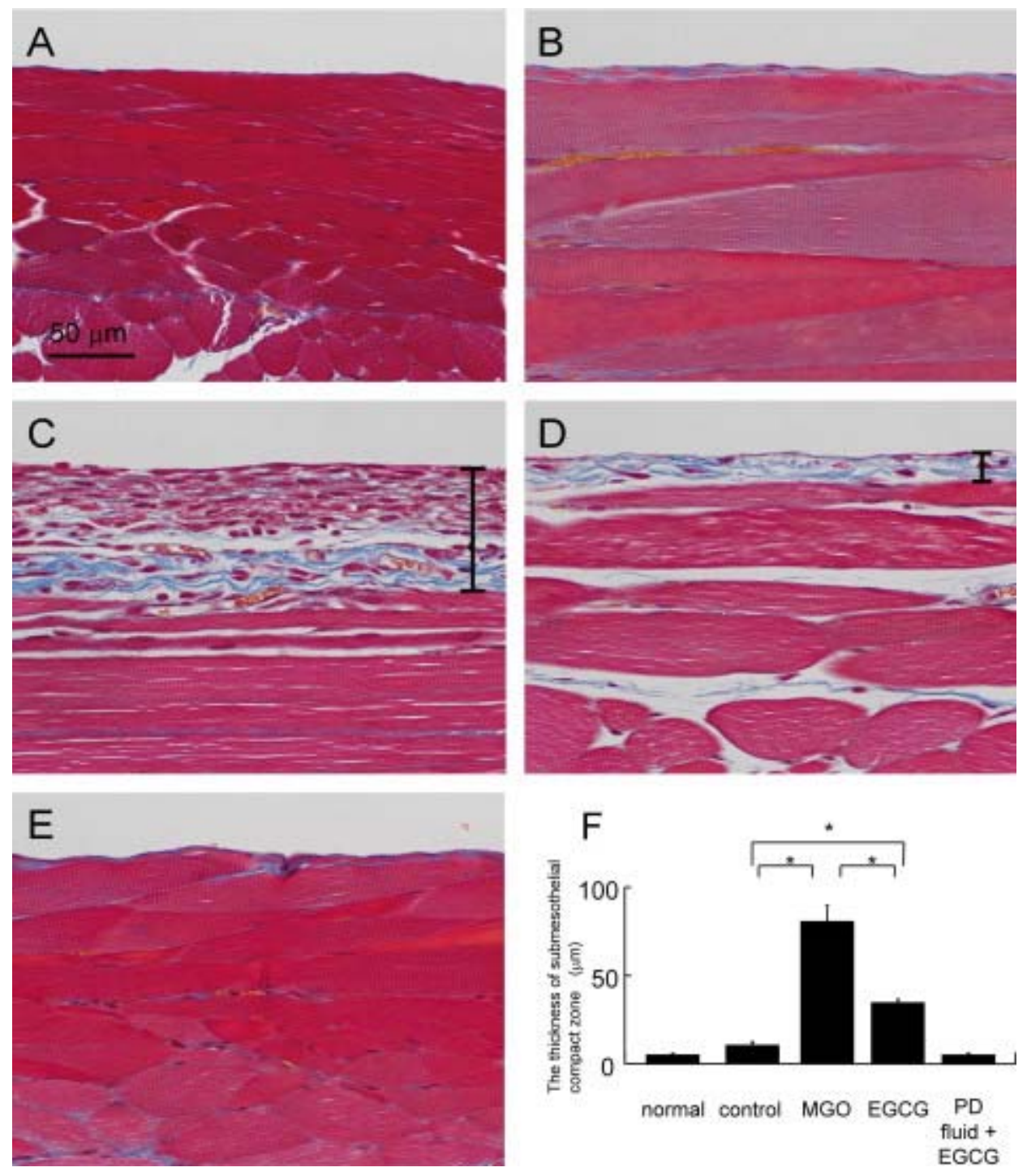

Fig. 1. Masson-trichrome staining of peritoneal tissues. (A) In normal mice, note the monolayer of mesothelial cells covered with the surface of peritoneum. (B) In the control group, injected with PD fluid and saline, the peritoneum is only slightly thickened. (C) MGO injection induced significant thickening of the peritoneum. (D) Administration of EGCG in MGO-treated mice significantly suppressed the progression of peritoneal thickening. (E) Repeated injections of EGCG had little effect on peritoneal thickening compared with the control group. (A-E), magnification $\times 200$; bars indicate the thickness of the submesothelial compact zone. (F) Bar graph showing the thickness of the submesothelial compact zone. Data are expressed as mean $\pm \mathrm{SEM} ;{ }^{*}$ represents $p<0.01$. 

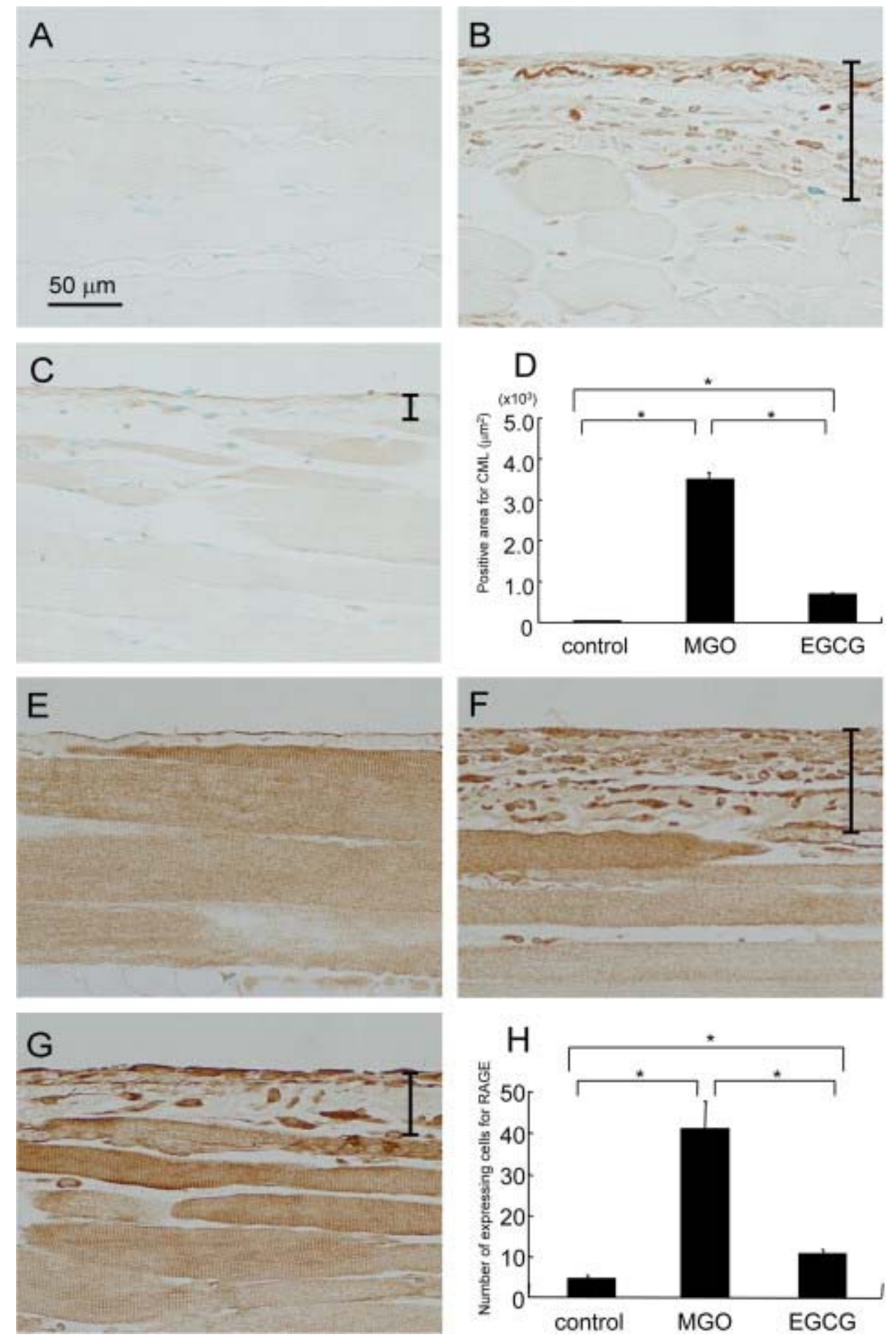

Fig. 2. The positive area of CML and the number of RAGE-expressing cells in

peritoneal tissues. (A) Note the low level of CML deposition in the peritoneal tissues of the control group. (B) MGO injection induced a significant increase in CML deposition in the thickened peritoneum. (C) In comparison with the MGO group, the EGCG group showed reduced intensity of CML staining in the peritoneum. (A-C) magnification, $\times 200$; bars indicate the thickness of the submesothelial compact zone. (D) Bar graph showing positive area of CML in the peritoneal tissues. Data are expressed as mean $\pm \mathrm{SEM} ;{ }^{*} p<0.01$. (E) 
Note the low level of RAGE expression in the control group. (F) MGO injection strongly increased RAGE expression in the submesothelial compact zone and the vascular wall. (G) In the EGCG group, the intensity of staining was significantly reduced. (E-G) magnification, $\times 200$; bars indicate the thickness of the submesothelial compact zone. $(\mathrm{H})$ Bar graph showing the number of RAGE-expressing cells. Data are expressed as mean $\pm \mathrm{SEM} ;{ }^{*} p<0.01$. 

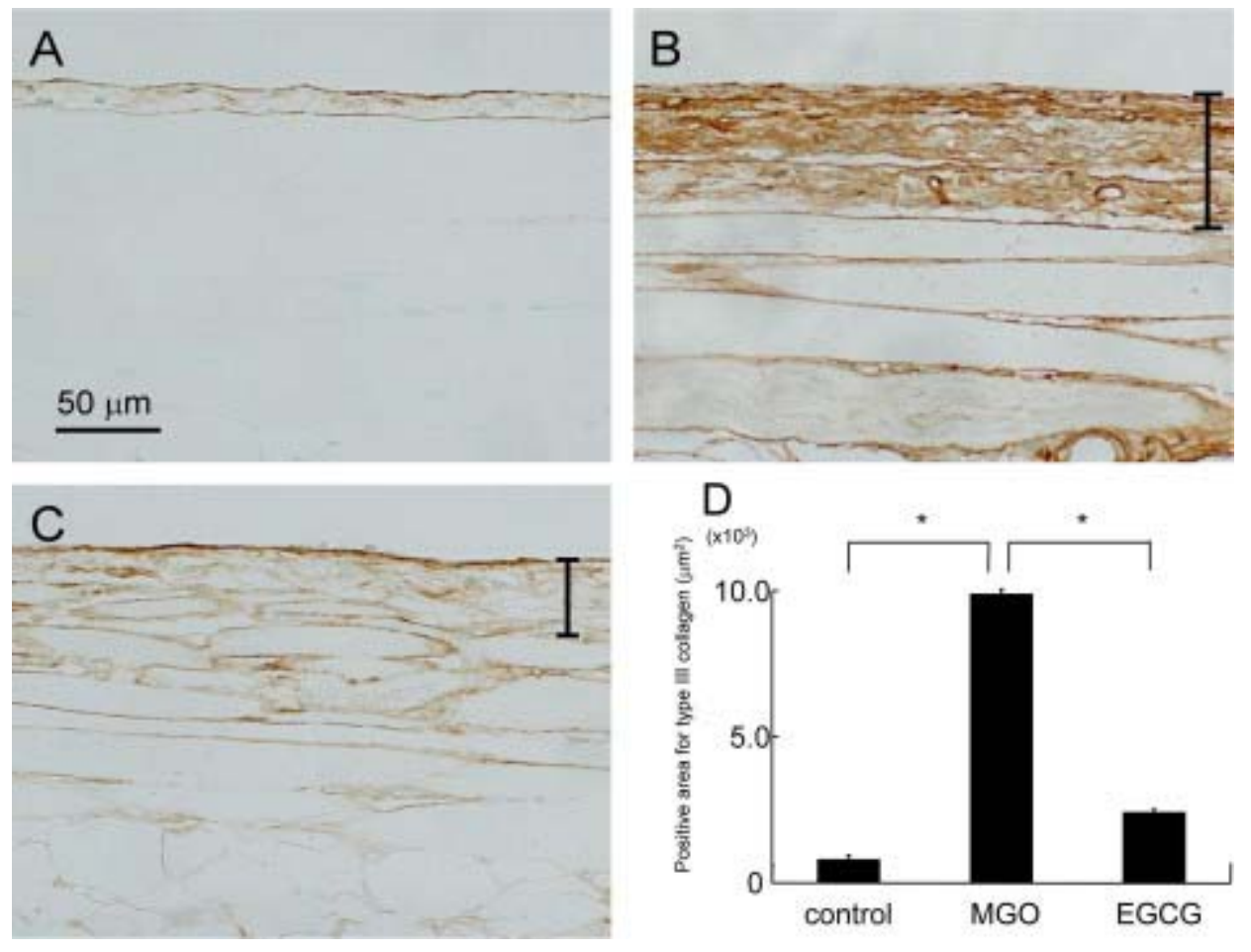

Fig. 3. The positive area of type III collagen in submesothelial compact zone. (A) In the control group, type III collagen expression in the submesothelial compact zone was equivalent to that of normal mice. (B) In the MGO group, the positive area of type III collagen in the submesothelial compact zone was significantly increased compared with the control group. (C) In comparison with the MGO group, the deposition of type III collagen in the EGCG group was significantly reduced (C). (A-C) magnification, $\times 200$; bars indicate the thickness of the submesothelial compact zone. (D) Bar graph showing the positive area of type III collagen in the submesothelial compact zone. Data are expressed as mean \pm SEM; * $p<0.01$. 

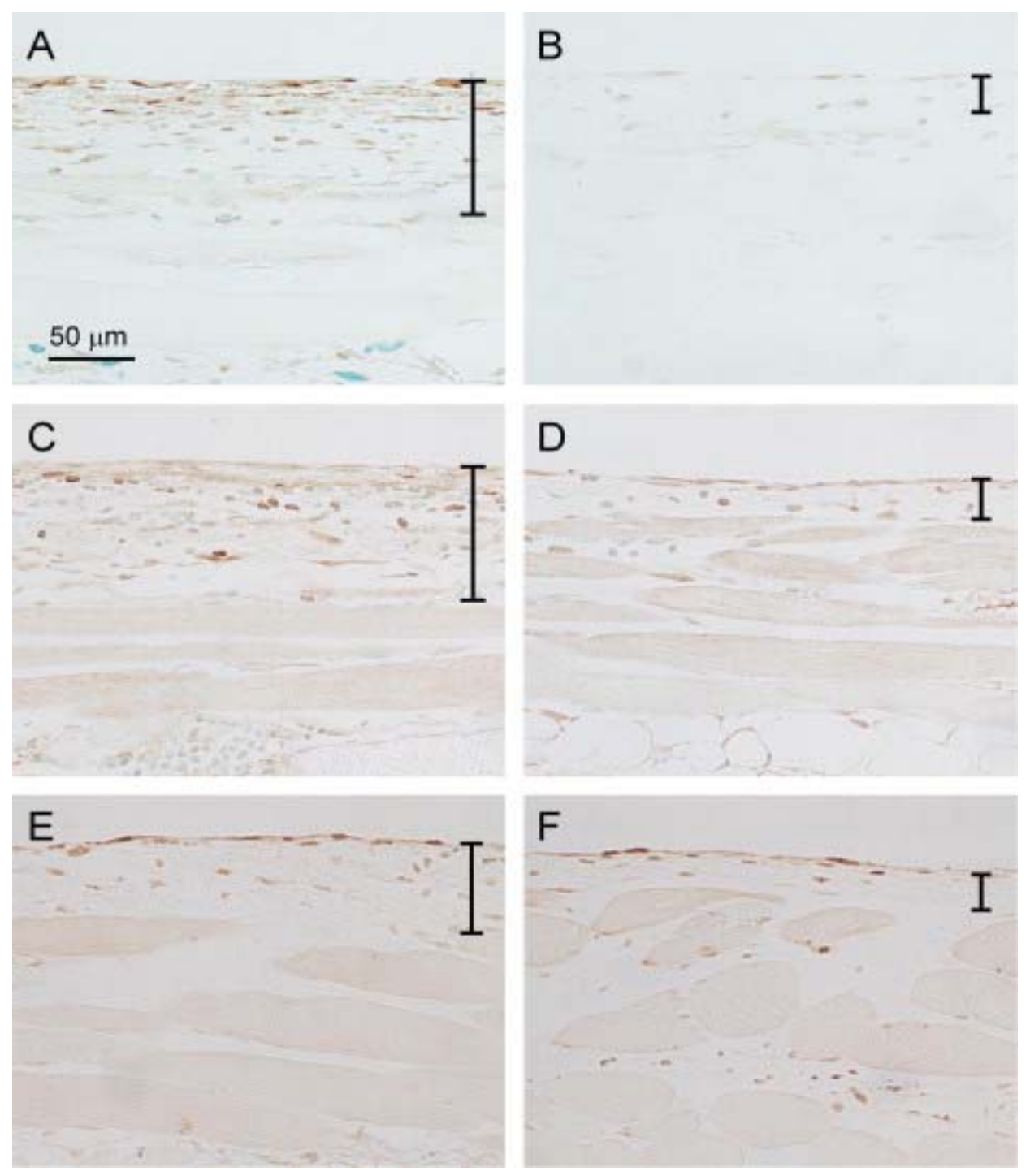

Fig. 4. Immunohistochemistry for $\boldsymbol{\alpha}$-SMA, TGF- $\boldsymbol{\beta}$, and 8-OHdG. (A) Note the presence of numerous $\alpha$-SMA-expressing cells in the thickened submesothelial compact zone of the MGO group. (B) In the EGCG group, the number of $\alpha$-SMA-expressing cells was reduced. (C) Note the strong expression of TGF- $\beta$ in the thickened submesothelial compact zone of the MGO group. (D) In the EGCG group, the expression of TGF- $\beta$ was decreased. (E) Note the strong expression of $8-\mathrm{OHdG}$ in the thickened submesothelial compact zone of the MGO group. (F) In the EGCG group, the expression of 8-OHdG was decreased. (A-F) magnification, $\times 200$; bars indicate the thickness of the submesothelial compact zone. 

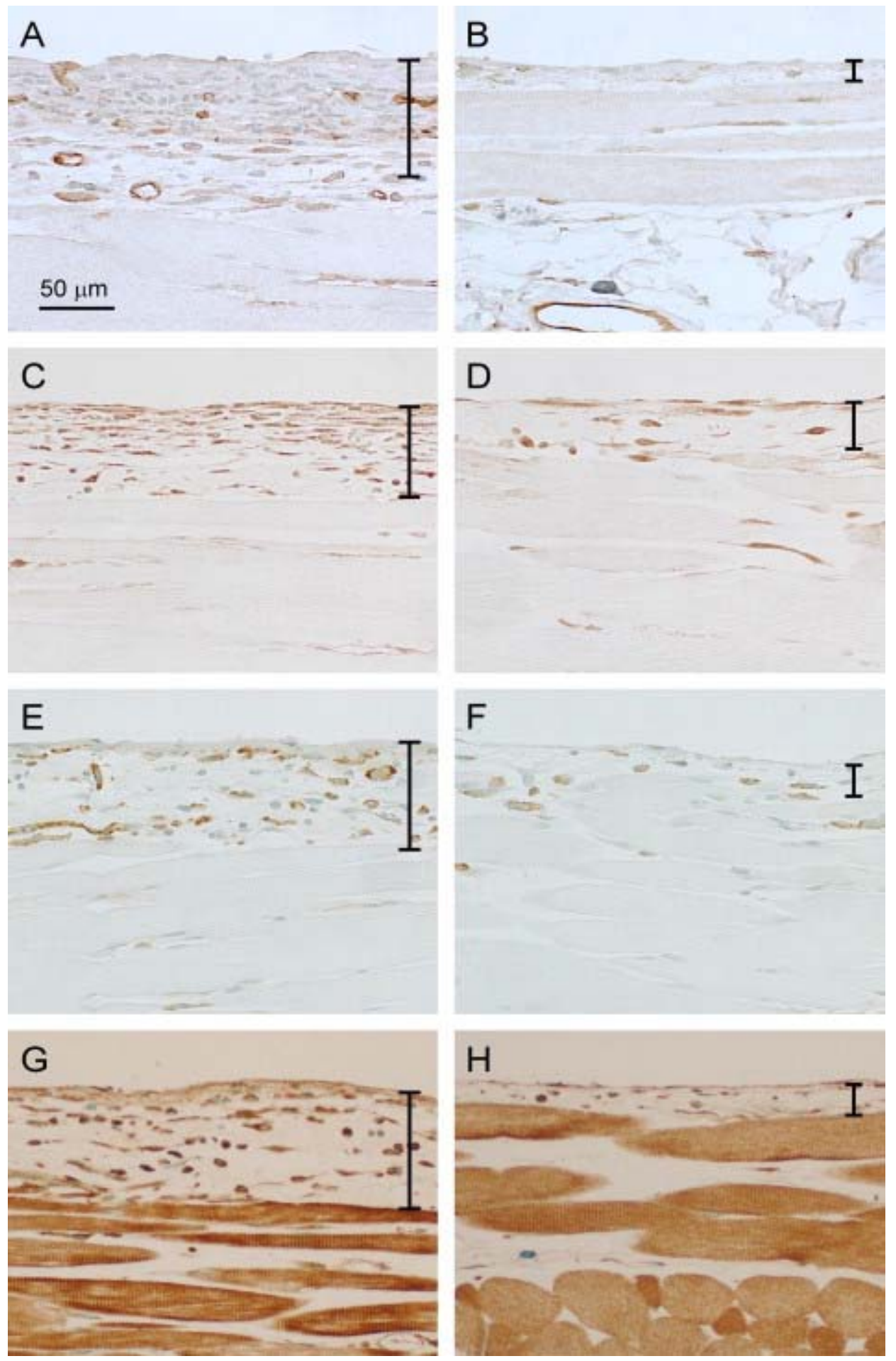

Fig. 5. Immunohistochemistry for CD31, VEGF, F4/80, and MCP-1. (A) Note the presence of numerous vessels positively stained for CD31 in the MGO group. (B) In the EGCG group, only a few vessels stained positively for CD31. (C) Note the large number of VEGF-expressing cells in the MGO group. (D) In the EGCG group, the number of VEGFexpressing cells was decreased. (E) F4/80-positive macrophages were present in thickened peritoneal tissues of the MGO group. (F) In the EGCG group, the number of F4/80-positive 
macrophages was reduced. (G) Note the strong expression of MCP-1-positive cells in the markedly thickened submesothelial compact zone of the MGO group. (H) In the EGCG group, the number of MCP-1-positive cells in the submesothelial compact zone was decreased. $(\mathrm{A}-\mathrm{H})$ magnification, $\times 200$; bars indicate the thickness of the submesothelial compact zone. 

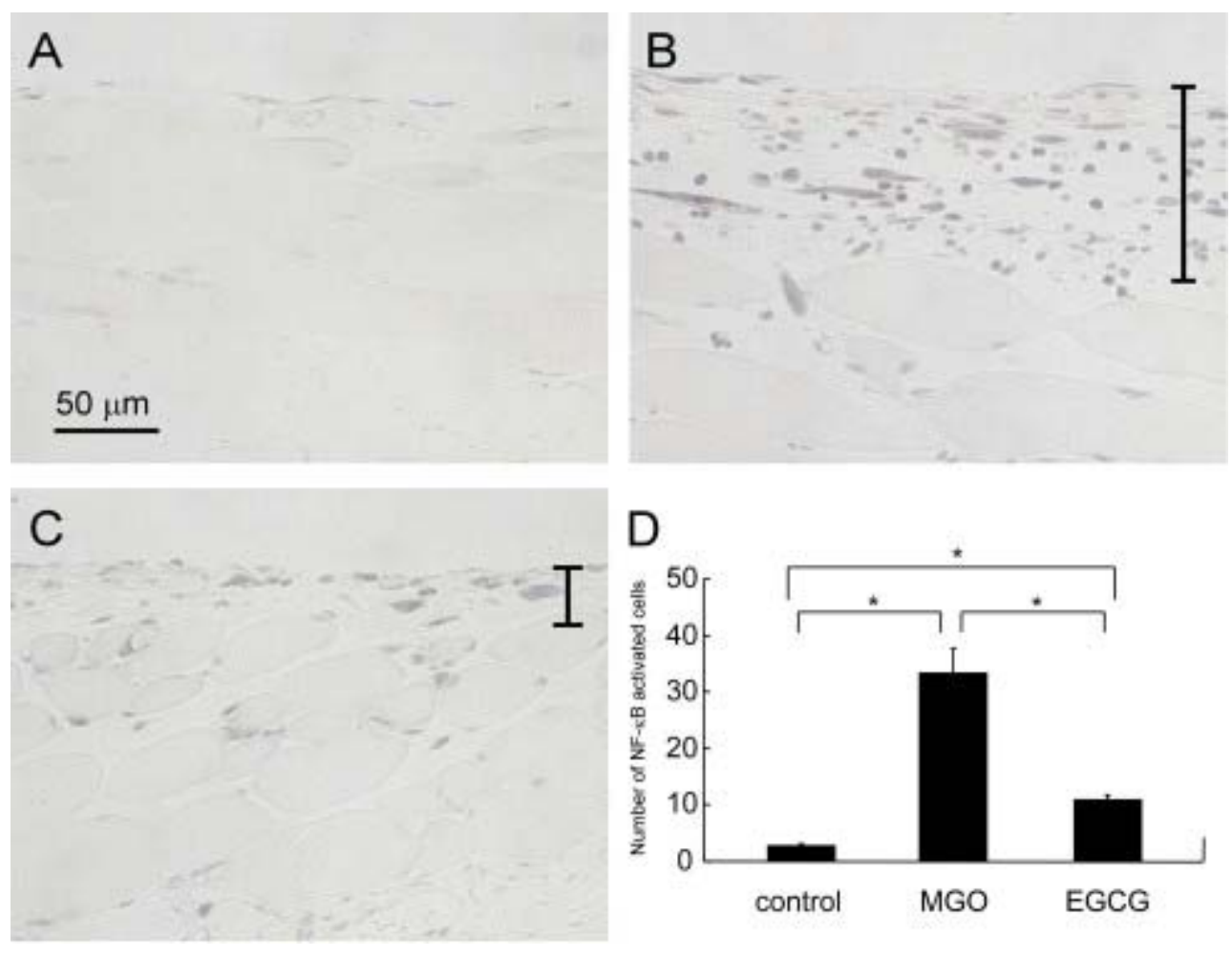

Fig. 6. Southwestern histochemistry for activated NF-кB. (A) In the control group, few NF- $\kappa \mathrm{B}$-activated cells were present in the mesothelial layer. (B) MGO injection induced a significant increase in the number of NF- $\mathrm{BB}$-activated cells in the submesothelial compact zone. (C) In comparison with the MGO group, the EGCG group contained significantly fewer NF- $\kappa \mathrm{B}$-activated cells in the submesothelial compact zone. (A-C) magnification, $\times 200$; bars indicate the thickness of the submesothelial compact zone. (D) Bar graph showing the number of NF- $\kappa \mathrm{B}$-activated cells in the submesothelial compact zone. Data are expressed as mean $\pm \mathrm{SEM} ; * p<0.01$. 
Table: Results of immunohistochemistry for TGF- $\beta, \alpha$-SMA, 8-OHdG, CD31, VEGF, F4/80, and MCP-1

Number of positive cells in the peritoneum

Control group $\quad$ MGO group EGCG group

\begin{tabular}{lrlrr} 
TGF- $\beta$ & 2.0 & \pm 0.66 & $28 \pm 3.2^{\mathrm{a}}$ & $12 \pm 0.98^{\mathrm{b}}$ \\
$\alpha-S M A$ & 0.22 & \pm 0.13 & $11 \pm 2.1^{\mathrm{a}}$ & $5.8 \pm 0.82^{\mathrm{b}}$ \\
8-OHdG & 6.1 & \pm 1.4 & $18 \pm 2.5^{\mathrm{a}}$ & $7.9 \pm 0.50^{\mathrm{b}}$ \\
CD31 & $0.10 \pm 0.08$ & $2.6 \pm 0.48^{\mathrm{a}}$ & $0.79 \pm 0.18^{\mathrm{b}}$ \\
VEGF & $1.3 \pm 0.25$ & $21 \pm 0.25^{\mathrm{a}}$ & $7.3 \pm 0.85^{\mathrm{b}}$ \\
F4/80 & $0.50 \pm 0.25$ & $13 \pm 1.7^{\mathrm{a}}$ & $5.7 \pm 0.49^{\mathrm{b}}$ \\
MCP-1 & $1.8 \pm 0.41$ & $18 \pm 3.2^{\mathrm{a}}$ & $6.6 \pm 0.81^{\mathrm{b}}$ \\
\hline
\end{tabular}

These data were determined in 5 fields of the submesothelial area selected at random in each mouse and examined at x 200 magnification. Values are given as mean \pm SEM

${ }^{a} \mathrm{p}<0.01$ vs. Control group.

${ }^{\mathrm{b}} \mathrm{p}<0.01$ vs. MGO group. 